\title{
Sobre dois novos nematodeos parasitos da quica: Caluromys philander (L.) *
}

\author{
por \\ Herman Lent e J. F. Teixeira de Freitas \\ (Com 10 figuras no texto)
}

\begin{abstract}
Neste trabalho descrevemos duas novas especies de helminthos nematodeos parasitos de uma quica não muito commum entre nós, capturada pelo Dr. J. C. N. Penido e determinada pelo Prof. Alipio Miranda Ribeiro, do Museu Nacional do Rio de Janeiro. Um delles pertence á superfamilia Spiruroidea Railliet \& Henry, 1915, familia Rictulariidae Railliet, 1916, genero Rictularia Froehlich, 1802 e é descripto sómente de 10 exemplares femeas já que não conseguimos encontrar nenhum exemplar macho; o outro pertence á superfamilia Subuluroidea Travassos, 1930, familia Subuluridae Yorke \& Maplestone, 1926, subfamilia Subulurinae Travassos, 1914, genero Subulura Molin, 1860 e é descripto de um unico exemplar macho.
\end{abstract}

Rictularia jaegerskioldi $\mathrm{n}$. $\mathrm{sp}$.

Comprimento:- Femea 16,648 a $24,617 \mathrm{~mm}$.

Largura maxima:- Femea 0,572 a $0,710 \mathrm{~mm}$.

Helminthos de côr branca, com corpo afilado nas extremidades, cuticula provida de estrias longitudinaes e transversaes, sendo estas muito pronunciadas e apresentando duas séries longitudinaes de formações chitinosas caracteristicas situadas ventro-lateralmente.

Essas formações se orginam ao nivel do inicio do esophago e terminam perto da extremidade posterior, em geral logo após o anus. São dispostas em numero de 80 a 82 pares e se differenciam em 2 typos principaes: o primeiro (fig. 1) é representado por formações que apresentam uma larga base estriada transversalmente e um pequeno aculeo dirigido para traz, e se situa do inicio do esophago até a região pre-vulvar; o segundo typo (fig. 2) é representado por verdadeiros espinhos, levemente encurvados, de base pequena é não estriada transversalmente, e se situa da região post-vulvar á extremidade posterior do corpo; entre esses 2 typos existem alguns pares de transição (fig. 3).

* Recebido para publicação a 31 de Julho de 1935. 


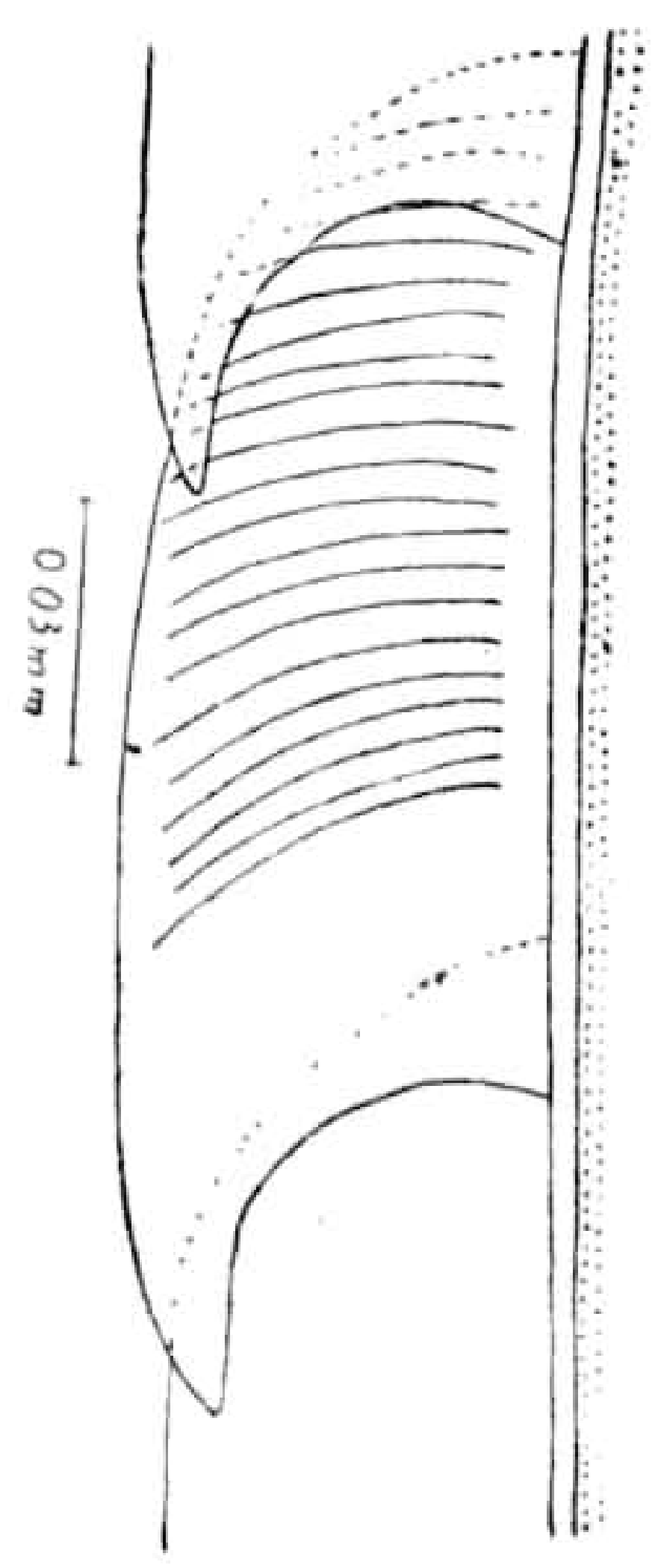

Fig. 1 - Rictularta jaegerskioldı n. sp. - Espinho cuticular.

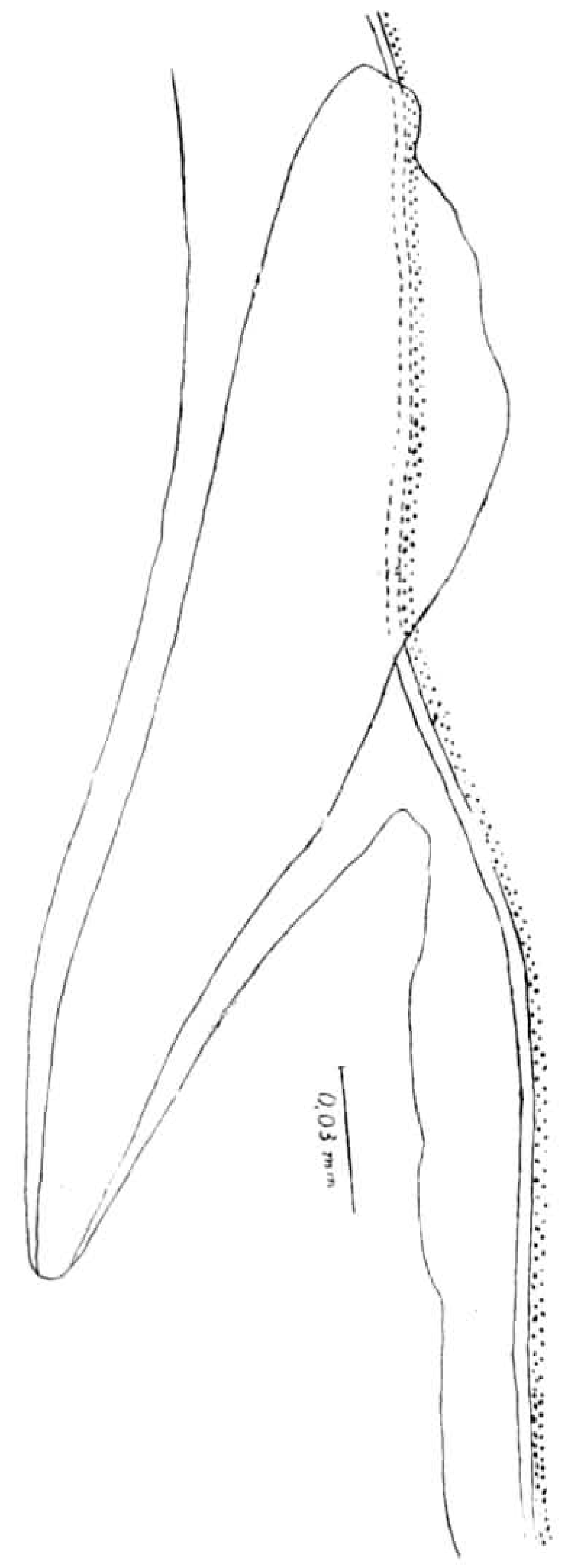

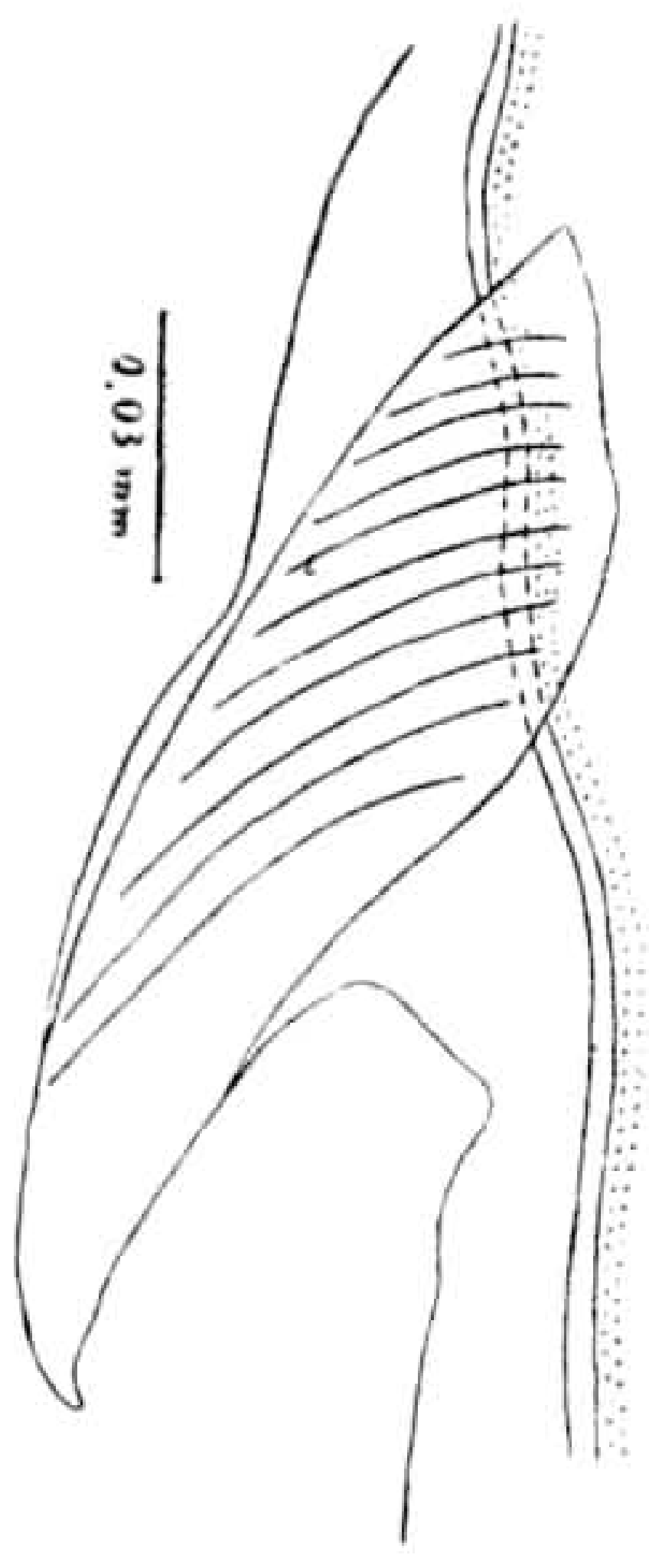

Fig. 3 - Rictularia jaegerskioldi $\mathrm{n} . \mathrm{sp}$.

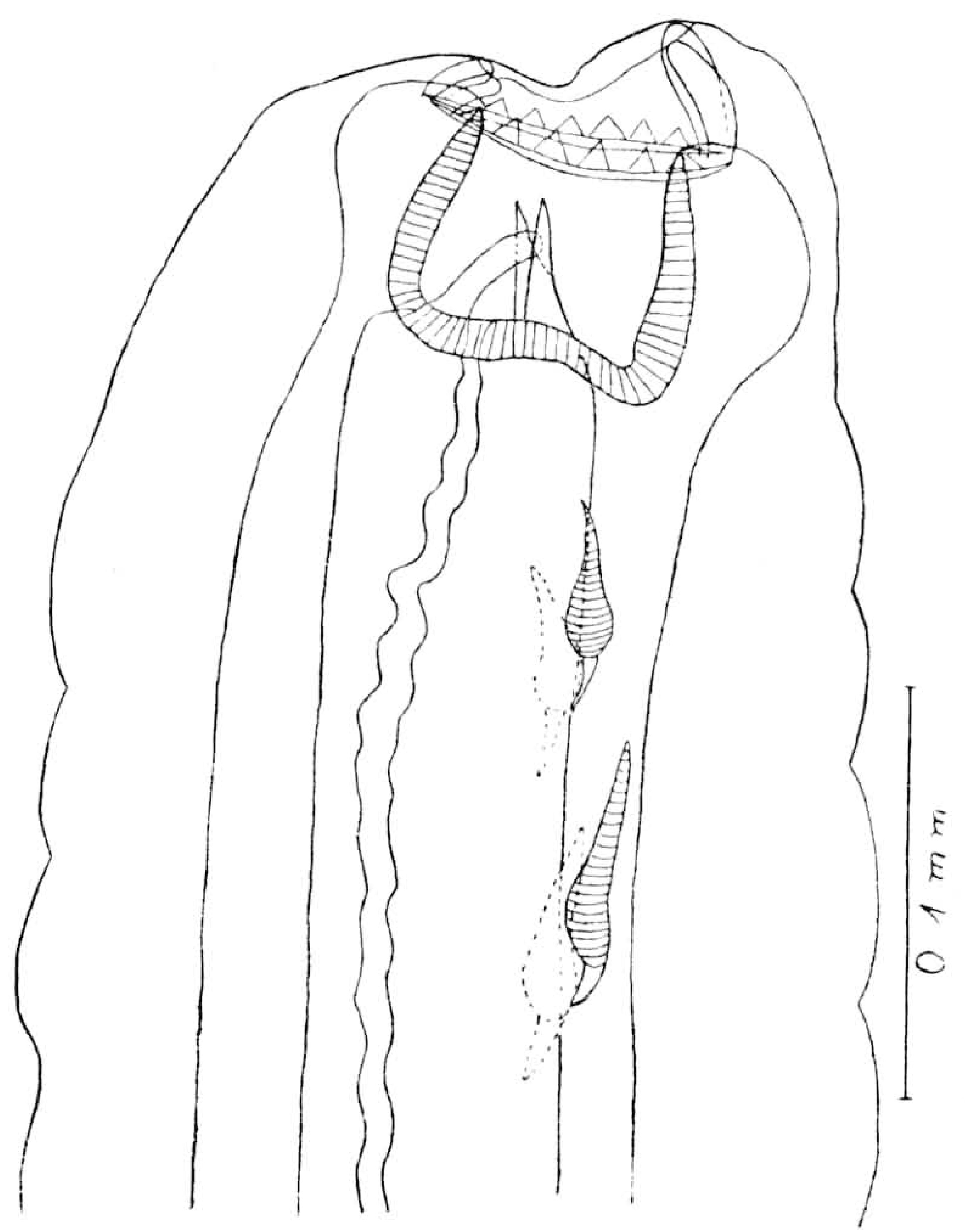

Fig. 4 - Rictularia jaegerskioldi n. sp. - Extremidade cephalica.

Fig. 2 - Rictularia jaegerskiold

n. sp. - Espinho cuticular.

As formações do 1.0 typo são muito proximas, havendo mesmo superposição em algumas dellas; á proporção que se caminha para a extremidade posterior do corpo ellas vão se afastando umas das outras cada vez mais.

A extremidade anterior apresenta um ampla capsula buccal (fig. 4) 
não desviada dorsalmente, medindo 0,056 a $0,080 \mathrm{~mm}$. de comprimento por 0,072 a $0,096 \mathrm{~mm}$. de largura, isto é, tendo secção mais ou menos quadrangular. A abertura buccal é circumdada por uma coronula de denticulos e por 2 pares de papillas, dos quaes um ventral, bem desenvolvido, e outro dorsal, menor. No fundo da capsula buccal observam-se 3 dentes, dos quaes dois ventraes, ponteagudos e rectos e um dorsal, de ponta romba, encurvado ventralmente e que geralmente termina entre os dois ventraes. Apresenta-se essa extremidade anterior cercada por uma culicula mais ou menos dilatada, o que é devido á fixação, pois que os exemplares vivos não apresentavam esta dilalação. Esophago longo, cylindrico, com 2,473 a 3,288 mm. de comprimento.

Vulva situada a cerca de 2,402 a $3,240 \mathrm{~mm}$. da extremidade anterior, abrindo-se no fundo de um forte sulco transversal, que se situa entre os 36.o

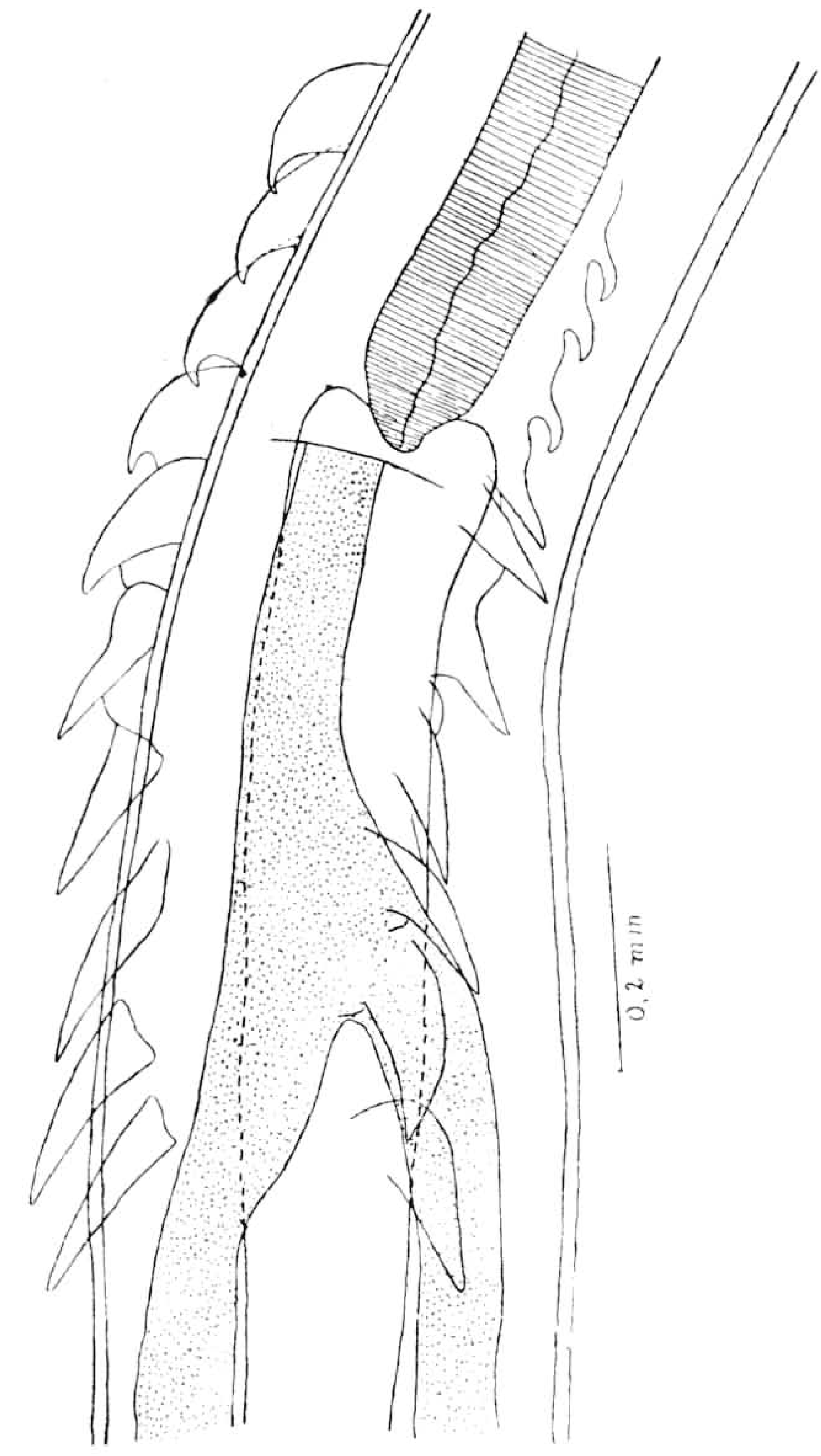

Fig. 5 - Rictularia jaegerskioldi n. sp. - Região vulvar; face ventral.

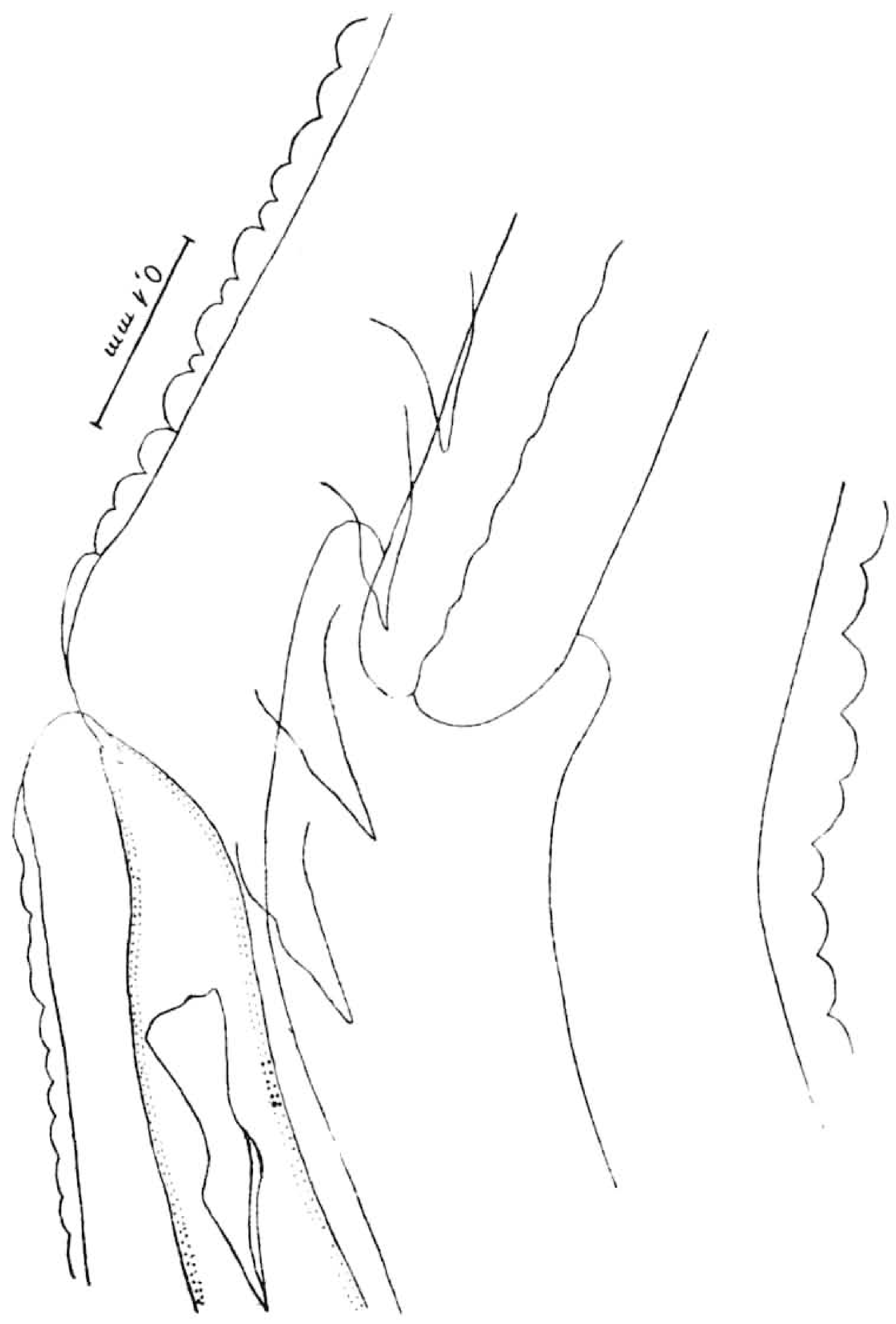

Fig. 6 - Rictularia jaegerskioldi n. sp. - Região vulvar, de perfil.

e 37.0 ou 38..$^{\circ}$ e 39..$^{\circ}$ pares de espinhos (figs. 5 e 6 ). Á ella segue-se um ovejector musculoso, impar até 0,280 a $0,658 \mathrm{~mm}$. para depois se bifurcar. Apparelho genital de typo prodelpho. Convem salientar que o ovejector possue, mesmo após fixação e clarificação pelo phenol, uma coloração amarella intensa. Ovos de casca espessa, lisa, com 0,038 a $0,046 \mathrm{~mm}$. de comprimento por 0,024 a $0,030 \mathrm{~mm}$. de largura. 


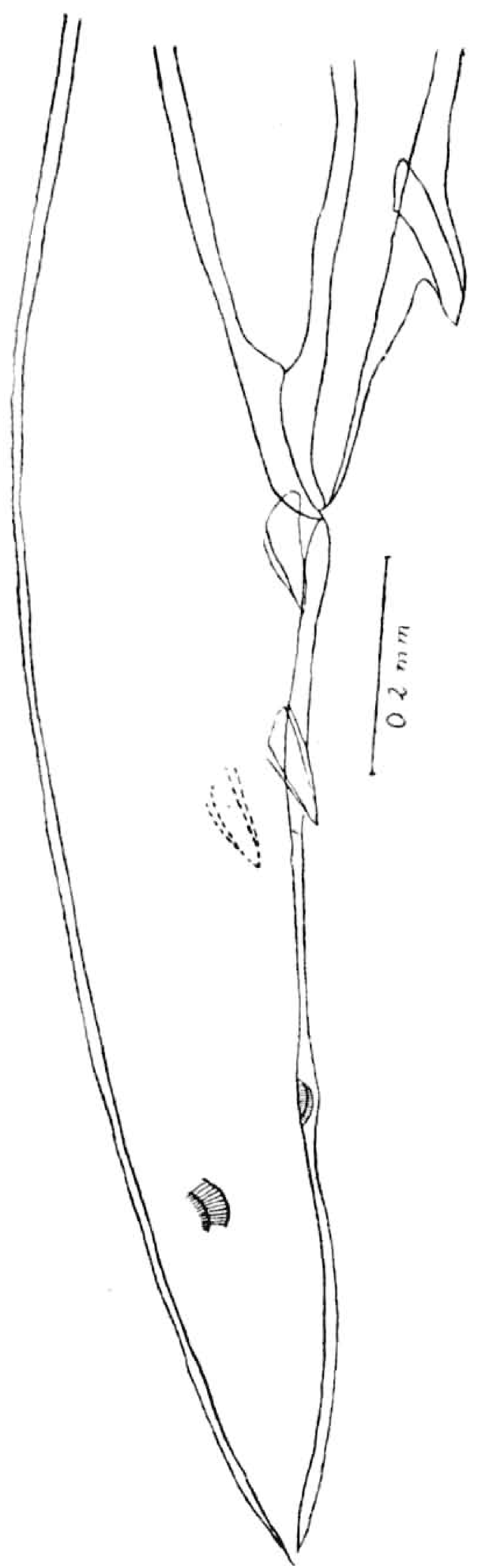

Fig. 7 - Rictularia jaegerskioldi n. sp. - Extremidade posterior da femea, de perfil.

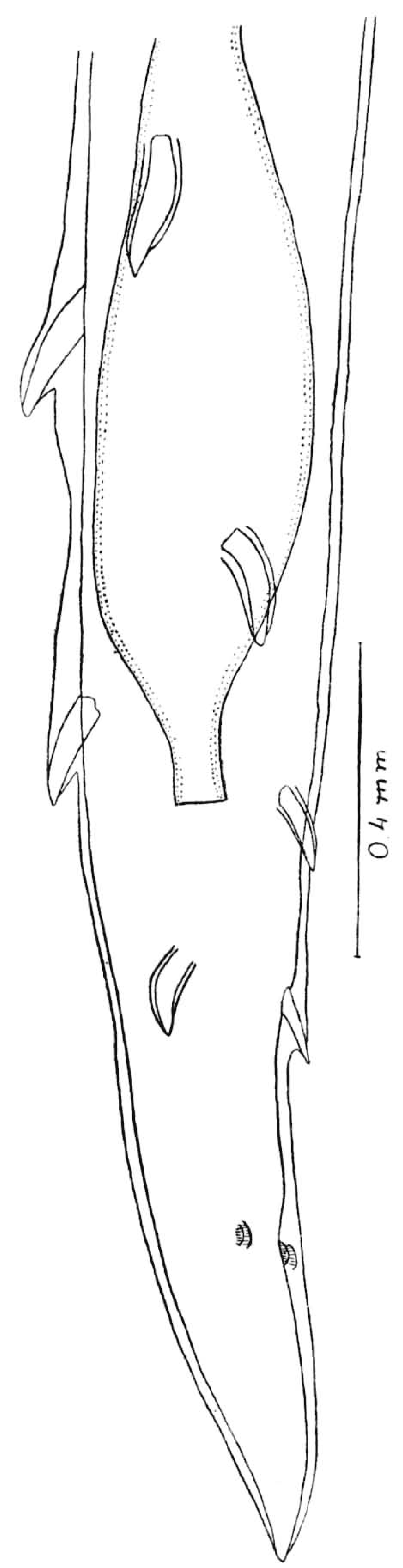

Fig. 8 - Rictularia jaegerskioldi 11. sp - Extremidade posterior da femea, de face.

Extremidade posterior conica. Anus (figs. 7 e 8 ) situado a cerca de 0,543 a $0,920 \mathrm{~mm}$. da cauda, precedido de um longo recto. Existe sempre um par de espinhos ad-anal. Atraz do anus observa-se uma certa variação no 
numero, na fórma e na situação dos espinhos, algumas vezes apenas esboçados, o que julgamos ser devido a um desenvolvimento retardado.

Habitat : - Intestino delgado de Caluromys philander (L.). Proveniencia : - Tijuca, Rio de Janeiro - Brasil.

Typos na collecção helminthologica do Instituto Oswaldo Cruz.

- Quem percorre a bibliographia existente sobre Rictularia logo se surprehende com o consideravel numero de especies descriptas sómente pelos exemplares femeas. Entretanto, é logo possivel verificar que isto tem acontecido devido á existencia de bons caracteres especificos nestes helminthos, como sejam: conformação e numiero dos espinhos latero-ventraes; fórma, estructura e situação da capsula buccal; comprimento do corpo e sua relação com o comprimento do esophago e da vulva; e mais, a especificidade parasitaria, que não póde deixar de ser levada em consideração.

Neste trabalho descrevemos uma nova especie, a primeira encontrada parasitando marsupial, que denominamos Rictularia jaegerskioldi em homenagem ao Prof. L. A. Jaegerskiold, autor da unica monographia publicada sobre o genero.

Para facilitar aos que se interessam pelo grupo organisamos uma lista das especies conhecidas e seus hospedadores:

R. cristata Froehlich, 1802 - em Mus sylvaticus.

R. plagiostoma (Wedl, 1861) - em Vespertilio mystacinus.

R. macdonaldi (Dobson, 1880) - em Megaderma frons.

R. bovieri R. Blanchard, 1886 -- em Vespertilio murinus.

R. elvirae Parona, 1889 - em Sciurus sp., Dremomys sp.

R. affinis Jaegerskiold, 1904 - em Vulpes niloticus.

R. cahirensis Jaegerskiold, 1904 - em gato e căo.

R. fallax Jaegerskiold, 1909 --- em Sciurus melanogaster.

R. splendida Hall, 1913 - em Canis nebracensıs.

$R$. proni Seurat, 1915 -.. em Herpestes sp.

R. coloradensis Hall, 1916 - em Futamias quadrivittatus.

R. disparalis Irvin-Smith, 1922 - em Hinulia sp.

$R$. amurensis Schulz, 1927 - em ratos.

$R$. caucasica Schulz, 1927 - em Gerbillus meridianus.

R. elegans Travassos, 1928 - em Eumusospes perotis.

R. mjoebergi Baylis, 1928 - em Arcticus binturong.

R. taterilli Baylis, 1928 -. em Taterillus gracilis angelus.

$R$. tani Hoepli, 1929 -- em Epimys norwegicus.

R. aethechini Le Roux, 1930 - em Aethechinus frontalis.

$R$. whartoni Tubangui, 1930 - em Mus norwegicus.

R. scalopis Goodrich, 1932 -- em Scalops aquaticus.

R. muris Galli-Valerio, 1932 - em ratos. 
R. citelli Mc Leod, 1933 - em Citellus tridecemlineatus e C. franklini.

R. whelleri Sandground, 1933 - em Paguma larvata intrudens.

R. alphi Lubimow, 1933 - em macacos.

R. sp. Baylis, 1934 - em Aethosciurus byatti.

R. $s p$. Mc Clure, 1934 - em Potos flavus.

R. harrisi Baylis, 1934 - em Mastomys coucha microdon.

R. halli Sandground, 1935 - em Eutamias striatus lysteri.

R. houdemeri Hsũ, 1935 - em Viverra zibetha.

Subulura interrogans $n$. $s p$.

Comprimento:- Macho 6,1 mm.

Largura maxima:- Macho $0,358 \mathrm{~mm}$.

Helmintho de côr branca, corpo rectilineo, afilado nas extremidades e provido de cuticula com delicadas estrias transversaes e numerosas estrias longitudinaes. Extremidade anterior apresenta duas azas cephalicas que medem $1,073 \mathrm{~mm}$. de comprimento por $0,057 \mathrm{~mm}$. de largura. Capsula buccal pequena, parecendo possuir dentes em sua base. Esophago com cerca de $1,073 \mathrm{~mm}$. de comprimento, isto é, terminando ao mesmo nivel das azas cephalicas; bulbo esophagiano com $0,200 \mathrm{~mm}$. de diametro.

Extremidade posterior com ventosa elliptica, distando $0,672 \mathrm{~mm}$. do fim do corpo. Azas caudaes pouco desenvolvidas. Papillas em numero de 12 pares, situadas do seguinte modo (figs. 9 e 10): 4 pares pre-anaes, 2 pares ad-anaes (embora estejam um pouco acima do orificio cloacal) e 6 pares postanaes. As papillas pre-anaes são dispostas, um par mais ou menos ao lado da parte mediana da ventosa, outro, de papillas pequenas, mais proximo do anus e 2 pares restantes intermediarios, mais proximos deste que daquelle, e constituidos de papillas de tamanhos desiguaes (um par pequeno e outro maior). Os pares de papillas ad-anaes são constituidos por 1 par de papillas maiores, alongadas e por outro par de papillas menores, arredondadas. Das papillas post-anaes 2 pares são de papillas arredondadas, medianas, 0 1.0 par um pouco maior que o $2 .^{\circ} ; 3$ pares são de papillas lateraes, proximas umas das outras, um par de papillas alongadas e volumosas, outro de papillas alongadas porém pequenas (finas) e o $3 .^{\circ}$ par de papillas arredondadas; por fim. encontra-se 1 par de papillas mais ou menos alongadas, tambem lateraes, situadas quasi na origem do espinho terminal da extremidade caudal. Esse espinho terminal mede $0,086 \mathrm{~mm}$. de comprimento. Anus situado a $0,172 \mathrm{~mm}$. da base do espinho terminal do corpo. Espiculos iguaes, bem chitinisados, com $1,144 \mathrm{~mm}$. de comprimento. Gubernaculo triangular, com sua porção proximal concava para a face ventral, medindo $0,168 \mathrm{~mm}$. de comprimento. 

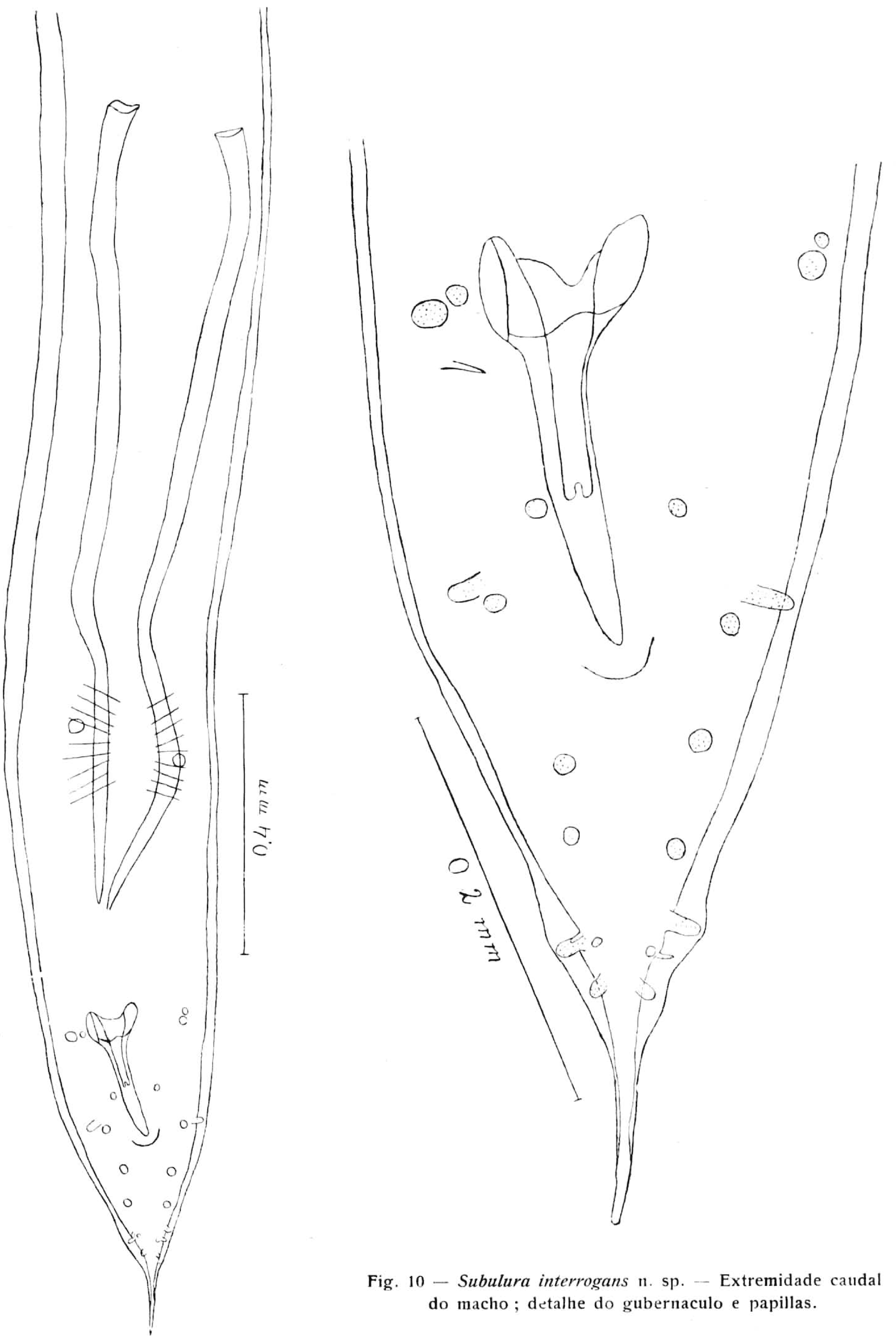

Fig. 10 - Subulura interrogans $\mathrm{n}$. sp. - Extremidade caudal do macho; detalhe do gubernaculo e papillas.

Fig. 9 - Subulura interrogans n. sp.Extremidade posterior do macho, mostrando os espiculos. 
Habitat:- Ceco de Caluromys philander (L).

Proveniencia : - Tijuca, Rio de Janeiro --- Brasil.

Typos na collecção helminthologica do Insiluto Oswaldo Cruz.

- Neste genero, que, entre os mammiferos, possue maior numero de especies parasitando primatas, encontramos uma unica especie descripta de um marsupial: - Subulura peramelis Baylis, 1930. A especie que acabamos de descrever della se differencia principalmente pelo numero de papillas caudaes ( 10 pares em $S$. peramelis) e pelo camprimento dos espiculos $(2,22 \mathrm{~mm}$. elm $S$. peramelis $)$, além de outros caracteres de mener importancia.

(Trabalho do Laboratorio de Helminthologia - Dr. Lauro Travassos). 